\title{
Radiofrequency ablation of small renal masses in comorbid patients
}

\author{
Jerzy Siekiera ${ }^{1}$, Milosz Jasinski ${ }^{1}$, Witold Mikołajczak ${ }^{2}$ \\ ${ }^{1}$ Department of Oncological Urology, Oncology Centre, Bydgoszcz, Poland \\ ${ }^{2}$ Department of Urology, Specialist Municipal Hospital, Torun, Poland
}

Videosurgery Miniinv 2018; 13 (2): 212-214

DOI: https://doi.org/10.5114/wiitm.2018.74462

\begin{abstract}
Introduction: Over the recent years, the progress in imaging techniques has led to increased detection of small renal masses (SRMs), including in elderly and high-risk patients. Partial nephrectomy (nephron-sparing surgery - NSS), the current standard of care in T1a kidney tumours, has some limitations in patients who are poor candidates for surgery, as it is associated with potential perioperative complications and possible renal function loss. Radiofrequency ablation (RFA), a minimally invasive method that can be performed percutaneously, is an option in such cases.

Aim: To present our experience in treatment of SRMs using RFA in comorbid patients.

Material and methods: In the years 2006-2012, 103 percutaneous, ultrasound-guided RFA procedures were performed in the Oncology Centre in Bydgoszcz in patients with an ASA score $\geq 3$. Abdominal computed tomography and tumour biopsy were performed before the procedure. The average follow-up time was 46 months.

Results: The 1, 3 and 5-year overall survival rates were respectively $97 \%, 90 \%$ and $75 \%$, while cancer-specific survival was $100 \%$. No Clavien-Dindo grade $\geq 3$ complications were observed.

Conclusions: Radiofrequency ablation performed percutaneously is a minimally invasive treatment and may be applied in patients who are, due to comorbidities, poor candidates for surgery. In comorbid patients, where other causes of death play an important role, the application of a minimally invasive treatment method with satisfactory oncological effectiveness is justified.
\end{abstract}

Key words: radiofrequency ablation, comorbidities, small renal masses.

\section{Introduction}

Over the recent years, the progress in imaging techniques and wide introduction of ultrasonography (US) and computed tomography (CT) imaging have led to increased detection of small renal masses (SRM, kidney tumours smaller than $4 \mathrm{~cm}$ ), including in elderly and high-risk patients [1, 2]. Partial nephrectomy (nephron-sparing surgery - NSS), the current standard of care in T1a kidney tumours, has some limitations in patients who are poor candidates for surgery, as it is associated with potential perioperative complications and possible renal function loss due to warm ischaemia [3-5].

Radiofrequency ablation (RFA), a minimally invasive treatment method that can be performed percutaneously, is increasingly used in treatment of SRMs, especially in elderly patients with comorbidities. It is associated with faster postoperative recovery and a low complication rate and may be an effective alternative for selected patients with SRMs $[3,6]$.

However, due to the lack of high quality data from randomized controlled trials, it is still debated 
whether RFA can achieve equivalent safety and longterm oncological efficacy.

\section{Aim}

The aim of this paper is to present our experience in treatment of SRMs using RFA in comorbid patients.

\section{Material and methods}

The retrospective analysis of medical records of patients who underwent RFA of kidney tumour in the years 2006-2012 in the Department of Oncological Urology, Oncology Centre in Bydgoszcz was performed. One hundred and three patients with comorbidities were included in the analysis. The inclusion criteria were ASA score $\geq 3$ (29 patients with ASA score 4, 74 ASA 3) and biopsy-proven T1a kidney cancer.

The average patients' age in this group was 68.6 years (51-89 years), and 19 patients were under 65 years. The average tumour diameter was $27.2 \mathrm{~mm}$ (10-40 mm). Eighteen patients had a tumour in a single kidney.

Tumours were imaged prior to RFA using contrast-enhanced $\mathrm{CT}$ or magnetic resonance (MR) US-guided biopsy was performed before RFA. Clear cell carcinoma was the dominating pathology - 98 cases, 3 chromophobe and 2 papillary cancer cases.

US-guided, percutaneous RFA was performed using alternating current $(460 \mathrm{kHz})$ conducted directly to the tumour with a needle electrode. The total ablation time was 8-30 min with 1-3 punctures, 8-12 min per puncture, depending on tumour diameter. In tumours under $25 \mathrm{~mm}$ only one puncture with an ablation time of 8-12 min was performed. Patients were followed according to the guidelines prepared for NSS - contrast-enhanced CT or MR 6, $12,24,36,48$ and 60 months after the procedure.

The information about patients' deaths was gathered from the Citizenship Department of Internal Affairs.

\section{Results}

Average follow-up time was 46 months (9-105 months). Seventeen patients died within 5 years after the procedure, but none because of kidney cancer. Causes of death were: respiratory insufficiency 7 , myocardial infarct -2 , stroke -2 , other neoplasm (not kidney) -2 , other -4 .
The 1-, 3- and 5-year overall survival (OS) rates were respectively 97\%, 90\% and 75\% (Figure 1).

The cancer-specific survival (CSS) was $100 \%$. The average hospital stay was less than 3 days and no Clavien-Dindo grade $\geq 3$ complications were observed.

\section{Discussion}

Over the last years, RFA has been gaining popularity and is now accepted as one of the treatment methods in SRMs [6-8]. Some authors report comparable results of RFA and NSS in selected patients $[3,9]$. It must also be stressed that percutaneous RFA is a minimally invasive treatment and can in certain cases be performed under local anaesthesia [6, 10]. It is associated with shorter hospital stay than NSS (even laparoscopic) and a low complication rate no serious (Clavien-Dindo grade $\geq 3$ ) complications were observed.

It has been questioned recently whether NSS, the current gold standard in treatment of SRMs, is still the best option in elderly and comorbid patients, with active surveillance (AS) becoming more accepted and used in such cases [11, 12]. Interestingly, Patel et al. reported lower OS and CSS in the non-surgical management group. The first phenomenon is easily understandable - patients qualified for non-surgical management had generally poorer health status, with substantial other-cause mortality. The second one, however, may suggest that the selection process for non-surgical management in older patients can be improved, as a significant proportion of them may still die of kidney cancer.

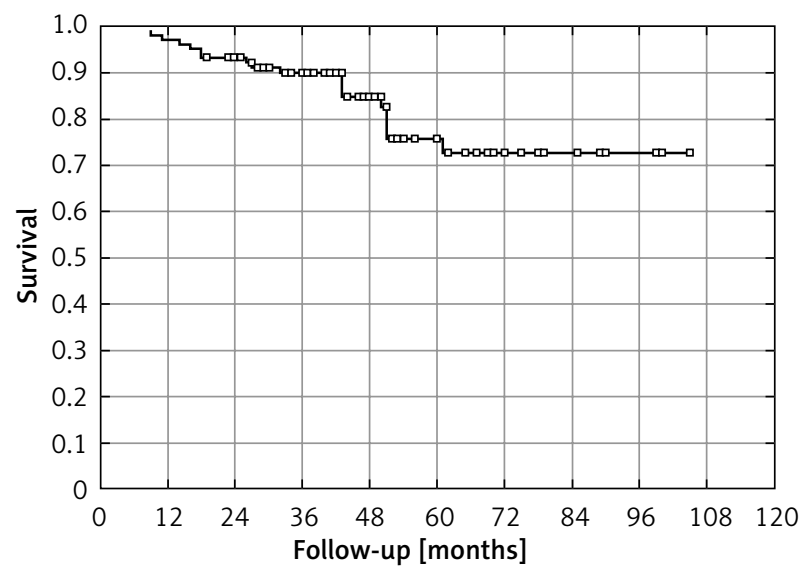

Figure 1. Overall survival after radiofrequency ablation 
It seems reasonable, then, that a subgroup of elderly patients with SRMs who are poor candidates for surgery may still benefit from a minimally invasive, focal treatment (such as RFA) in comparison to AS. The CSS rate observed in our material is comparable to CSS reported by Patel et al. in the NSS group, and significantly higher than CSS reported for the non-surgical management group [11]. The significant other-cause mortality should be attributed to the generally poor health status of the patients qualified for RFA in our study.

Another issue worth considering is the economic aspect of treatment of SRMs - the short hospital stay and low complication rate of percutaneous RFA should translate into lower cost of treatment [13].

This paper has limitations, mainly the lack of a proper, preferably randomized, control group. It is uncertain, however, whether a randomized trial RFA versus AS should be performed, given the presence of evidence suggesting a possible survival benefit for at least some of the patients. Non-randomized control groups, on the other hand, suffer from inevitable selection bias, with a tendency to assign patients with poorer health status to AS.

Nevertheless, further effort is required to elucidate the qualification criteria for NSS, focal ablative treatment and AS in elderly, comorbid patients, including oncological effectiveness, risk of complications, risk of cancer progression and life expectancy.

\section{Conclusions}

Radiofrequency ablation performed percutaneously is a minimally invasive treatment and may be applied in patients who are, due to comorbidities, poor candidates for surgery.

In comorbid patients, where other causes of death play an important role, the application of a minimally invasive treatment method with satisfactory oncological effectiveness is justified.

\section{Conflict of interest}

The authors declare no conflict of interest.

\section{References}

1. Miller DC, Ruterbusch J, Colt JS, et al. Contemporary clinical epidemiology of renal cell carcinoma: insight from a population based case-control study. J Urol 2010; 184: 2254-8.

2. Rini BI, Campbell SC, Escudier B. Renal cell carcinoma. Lancet 2009; 373: 1119-32
3. Yin X, Cui L, Li F, et al. Radiofrequency ablation versus partial nephrectomy in treating small renal tumors: a systematic review and meta-analysis. Medicine (Baltimore) 2015; 94: e2255.

4. Pan XW, Cui XM, Huang H, et al. Radiofrequency ablation versus partial nephrectomy for treatment of renal masses: a systematic review and meta-analysis. Kaohsiung J Med Sci 2015; 31: 649-58.

5. Sönmez MG, Kara C. The effect of zero-ischaemia laparoscopic minimally invasive partial nephrectomy using the modified sequential preplaced suture renorrhaphy technique on long-term renal functions. Videosurgery Miniinv 2017; 12: 257-63.

6. Jasinski M, Siekiera J, Chlosta P, et al. Radiofrequency ablation of small renal masses as an alternative to nephron-sparing surgery: preliminary results. Videosurgery Miniinv 2011; 6: 242-5.

7. Wagstaff P, Ingels A, Zondervan P, et al. Thermal ablation in renal cell carcinoma management: a comprehensive review. Curr Opin Urol 2014; 24: 474-82.

8. Ljungberg B, Bensalah K, Canfield S, et al. EAU guidelines on renal cell carcinoma: 2014 update. Eur Urol 2015; 67: 913-24.

9. Georgiades CS, Rodriguez R. Renal tumor ablation. Tech Vasc Interventional Rad 2013; 16: 230-8.

10. Ginzburg S, Tomaszewski JJ, Kutikov A. Focal ablation therapy for renal cancer in the era of active surveillance and minimally invasive partial nephrectomy. Nat Rev Urol 2017; 14: 669-82.

11. Patel HD, Kates M, Pierorazio PM, et al. Survival following diagnosis of localized T1a kidney cancer: current population-based practice of surgery and nonsurgical management. Urology 2014; 83: 126-33.

12. Mehralivand S, Neisius A, Thomas C, et al. Treatment of CT1a renal tumours in Germany: a nationwide survey. Urol Int 2016; 96: $337-44$

13. Castle SM, Gorbatiy V, Avallone MA, et al. Cost comparison of nephron-sparing treatments for CT1a renal masses. Urol Oncol 2013; 31: 1327-32.

Received: 27.11.2017, accepted: 20.01.2018. 